\title{
A POLÍTICA DE REFORÇO DA RESPOSTA DOS CUIDADOS DE SAÚDE PRIMÁRIOS EM PORTUGAL: \\ PSICOLOGIA, OFTALMOLOGIA, OBSTETRÍCIA, PEDIATRIA E MEDICINA FÍSICA E DE REABILITAÇÃO
}

Denise Capela dos Santos

ORCID ID:0000-0002-5794-5203;

CIÊNCIA ID: 0B10-1C58-71EB,

dsantos@autonoma.pt

Os Cuidados de Saúde Primários (CSP) representam o primeiro nível de contato da comunidade com o Serviço Nacional de Saúde (SNS), assegurando, de forma personalizada e próxima, a educação para a saúde, a prevenção da doença, o diagnóstico, o tratamento e a reabilitação.

Em Fevereiro de 2016, o Ministro da Saúde lançou o "Plano Estratégico para a Reforma dos Cuidados de Saúde Primários", conferindo importância e prioridade à Reforma dos Cuidados de Saúde Primários que se tinha iniciado em 2005 (República Portuguesa, 2016) ${ }^{\text {d. Esta reforma }}$ inclui a contratação de médicos de Medicina Geral e Familiar (MGF) para que todos os cidadãos possam ter médico de família, baseia-se na melhoria da qualidade dos CSP e na expansão das Unidades de Saúde Familiares, por proximidade e colaboração estreita com os Hospitais (Augusto e Fronteira) ${ }^{\mathrm{a}}$.

No mês de Outubro deste ano, foi apresentado o Orçamento de Estado (OE) 2017. Este OE estabelece como prioridades para a saúde a redução das desigualdades no acesso aos serviços de saúde e a melhoria da capacidade da Rede de Cuidados de Saúde Primários.

Para dar resposta a estes objetivos, o governo define como diretrizes de ação:

1) A abertura de concursos para o preenchimento de vagas na área da MGF,

2) O alargamento da intervenção das Equipas de Saúde Familiar,

3) A abertura de novas Unidades de Saúde Familiar (USF), e

4) O reforço da capacidade dos CSP relativamente ao acesso a Meios Complementares de Diagnóstico e Terapêutica (MCDT) (República Portuguesa, 2016)a.

Este compromisso assumido pelo Governo sugere que existirá, no próximo ano, um aumento de recursos financeiros, humanos, de equipamentos, de materiais e de instalações, nos CSP, que passarão assim a ter uma maior capacidade de resposta. É importante contudo que este reforço de recursos chegue ao acompanhamento psicológico na doença mental por psicólogos, ao 
acompanhamento da saúde visual da população por oftalmologistas, ao acompanhamento de grávidas e de crianças até aos 18 anos de idade pela ginecologia/ obstetrícia e pediatria, respetivamente, e à reabilitação de pessoas com incapacidade física por fisioterapeutas e fisiatras. Para isso, é preciso que existam salas disponíveis, com equipamentos e materiais adequados ao diagnóstico e ao tratamento necessário, assim como técnicos de saúde habilitados para o efeito.

\section{AUMENTO DE RECURSOS FINANCEIROS NOS CSP}

O OE 2017 prevê um aumento da despesa com as ARS, que financiam os CSP. Realmente, este Orçamento de Estado propõe um aumento da despesa total consolidada de Serviços e Fundos Autónomos de 25,2\% face a 2016 (República Portuguesa, 2016)a. Nos Serviços e Fundos Autónomos estão incluídos: a Administração Central do Sistema de Saúde (ACSS), as Administrações Regionais de Saúde (ARS), Hospitais SPA (Setor Público Administrativo), ACES, ULS SPA, INEM, INFARMED, Entidade Reguladora da Saúde (ERS), Instituto Nacional Doutor Ricardo Jorge (INSA) e a Empresa Ambiente na Saúde (EAS). O valor de despesa contemplado no OE com estes serviços ascende a 44\% do total da despesa em saúde prevista para 2017 (República Portuguesa, 2016)a .

Por análise do MAPA VII, de Despesas dos Serviços e Fundos Autónomos, por classificação orgânica, com especificação das despesas globais de cada serviço e fundo, pode-se constatar que se prevê uma maior despesa para a ACSS e para todas as ARS no OE 2017, relativamente a 2016. Estes dados sugerem que o Governo planeia investir mais dinheiro na prestação de CSP. Esta vontade política expressa no OE é coerente com os objetivos previamente estabelecidos para a reforma das CSP. É no entanto necessário que este dinheiro chegue aos ACES e ULS e que seja alocado ao aumento da capacidade de resposta das Unidades Funcionais de prestação de CSP em áreas como a psicologia, a oftalmologia, a obstetrícia, a pediatria e a medicina física e de reabilitação.

De forma a potenciar o reforço das capacidades dos CSP para as áreas citadas, o Governo poderia apostar no controlo de metas relativas a indicadores de desempenho associados a cada uma destas áreas. Sugere-se portanto realizar o estudo periódico, trimestral, de:

1) O que cada ACES ou ULS recebeu em termos financeiros, da ARS respetiva;

2) O montante que gastou na compra de equipamentos, material e na contratação de recursos humanos, por especialidade;

3) O número de equipamentos e de material que comprou e o número de profissionais de saúde de diferentes classes que contratou, por Unidade Funcional e por especialidade, por 100.000 habitantes;

É importante avaliarmos estes dados face à:

4) Média de pacientes atendidos, por categoria profissional, por especialidade; 
5) Número de vezes que se utilizaram os equipamentos ou materiais recentemente adquiridos, por Unidade Funcional.

Idealmente, poder-se-ia também avaliar, por especialidade e por Unidade Funcional:

6) A satisfação da população com o serviço prestado;

7) O tempo de espera para acesso à primeira consulta;

8) O tempo de espera entre práticas clínicas para os pacientes em acompanhamento, por patologia.

Esta informação deveria ser recolhida por cada ACES/ ULS, agregada pela ARS correspondente e compilada pela ACSS, para controlo financeiro e político, pelo gabinete do Ministro.

Considera-se que o aumento de despesa considerada no OE 2017 é compatível com a sustentabilidade financeira do Serviço Nacional de Saúde (SNS), assumida publicamente pelo Governo e que todos ambicionamos. É fundamental que este aumento da despesa previsto com o SNS, no OE, sirva o interesse dos Portugueses relativo ao aumento da capacidade de resposta do serviço, por aumento de recursos disponíveis, e não para cobrir o incremento da dívida passada. De notar que, de acordo com os dados do Relatório de Execução Orçamental do 1ำ Semestre de 2016, a dívida do SNS a fornecedores aumenta cerca de 1.000 .000 de euros por dia, colocando dúvidas sobre o alcance das metas financeiras do SNS até ao final do ano, e o cumprimento dos objetivos definidos no Orçamento de Estado de 2016.

No entanto, referindo-se aos resultados de Abril da conta do SNS, o Ministro assume que «indicam um défice de 99,8 milhões de euros, um valor melhor que no mesmo período do ano anterior em 77 milhões de euros» (República Portuguesa, 2016)i.

\section{AUMENTO DE RECURSOS HUMANOS NOS CSP}

No início do seu mandato, o Governo prometeu uma redução considerável do número de Portugueses sem médico de família e o Ministro Adalberto Campos Fernandes tem vindo a reafirmar esta intenção nalgumas conferências de imprensa, onde anunciou que era sua pretensão "chegar ao final de 2016 com pouco mais de 500 mil portugueses sem médico de família, quando em Novembro de 2015, havia quase 1,2 milhões" (República Portuguesa, 2016) ${ }^{c}$. De acordo com o Ministro, para suprir as necessidades de todos os utentes dos Cuidados de Saúde Primários seriam precisos mais 616 médicos de MGF (República Portuguesa, 2016)g.

Esta medida traria uma grande mais-valia à saúde da população, facilitando ao acesso aos CSP. Contudo, ficam algumas dúvidas quando à sua capacidade de financiamento por parte do Governo e quanto à disponibilidade de médicos de MGF no território Português.

De facto, nos termos do disposto no artigo 28.. da Lei n.․ 7-A/2016, de 30 de Março, em conjugação com o artigo 94.. do Decreto-Lei n.. 18/2016, de 13 de Abril, "a celebração ou renovação de contratos de trabalho pelas unidades locais de saúde com a natureza de entidade 
pública empresarial integrados no Serviço Nacional de Saúde, depende de prévia autorização do membro do Governo responsável pela área da saúde" (ACSS, 2016)a․ A publicação desta Lei sugere que poderá não haver capacidade financeira para alcance da meta do Governo, trazendo uma barreira ao reforço das capacidades dos cuidados de saúde primários, que ninguém pretendia.

No entanto, contrariando esta ideia, em Junho de 2016, o Ministério da Saúde realizou um concurso público com 338 vagas de diversas unidades de cuidados primários: 68 do Norte, 54 do Centro, 175 em Lisboa e Vale do Tejo, 11 no Alentejo e 30 no Algarve, no seguimento do Plano estratégico de Reforma dos Cuidados Primários (Augusto, Simões e Fronteira, 2016). Em Setembro, só 276 tinham terminado o processo de candidatura, com escolha das suas preferências por ACES / ULS. Pela primeira vez este processo foi simplificado e não se procederam a entrevistas, com vista à colocação dos médicos mais rapidamente no terreno (Decreto-Lei n. $.24 / 2016$, de 8 de Junho) (Augusto, Simões e Fronteira, 2016).

Fica o apontamento de que, embora positivo para maior rapidez na colocação de médicos ao serviço da comunidade, redução das listas de espera e maior acessibilidade aos CSP, este procedimento contempla um grande risco, uma vez que competências técnicas no diagnóstico e prescrição do tratamento adequado, garantidas pela inscrição na Ordem dos Médicos, não representam competências emocionais, essenciais ao bom desempenho do médico junto dos pacientes e à boa integração na equipa de trabalho. Este tipo de competências só se conseguem avaliar num processo de seleção por entrevista. Se é verdade que em períodos de carência de profissionais os critérios de seleção não são rígidos, considera-se que é importante manter o processo, uma vez que se pretendem contratar médicos que gerem empatia com os doentes, que saibam e que os queiram ouvir, que saibam comunicar e que tenham uma palavra positiva a dizer a uma franja da população fragilizada. Correremos o risco de ter um atendimento mais rápido e de menor qualidade? Será esta a melhor solução?

O Governo implementou outras medidas para solucionar este problema.

Recorreu-se ao recrutamento de médicos MGF já reformados para o SNS (Augusto e Fronteira, 2006) a e aumentaram-se as vagas da especialidades de MGF (com 473 internos, em 2016) (República Portuguesa, 2016) ${ }^{\mathrm{e}}$

É também frequente haver anúncios de escassez de médicos de MGF em determinadas zonas do país mais periféricas (Algarve e Alentejo, por exemplo) e principalmente quando a deslocação dos médicos não é sujeita a recompensa financeira. Atento a este problema, o Governo aprovou os termos e condições da atribuição de incentivos à mobilidade geográfica de médicos e fixação em regiões menos favorecidas (República Portuguesa, 2016)c. Fica por avaliar o sucesso desta iniciativa, uma vez que não se encontraram dados sobre o número vagas abertas por concurso públicos e o número de contratos assinados pela MGF, nas Unidades Funcionais pertencentes aos ACES e ULS com menor número de médicos por 100.000 habitantes. 
$\mathrm{Na}$ tentativa de colmatar a falta de médicos no Algarve, Adalberto Campos Fernandes optou por realizar uma parceria com o Ministro da Ciência, Tecnologia e Ensino Superior para a criação do Centro Académico de Investigação e Formação Biomédica do Algarve, como forma de motivar os médicos a ficarem na região (República Portuguesa, 2016) . Esta ideia considera-se inovadora e a reproduzir. A classe médica é conhecida por gostar de inovar e de realizar investigação que contribua para o avanço da medicina. Contudo, depara-se muitas vezes com barreiras ao financiamento das suas iniciativas pela gestão das organizações, que, quando sujeitas ao subfinanciamento pelo Estado para cumprimento da sua missão, não têm verba para adjudicar em função do desenvolvimento da Ciência.

Outra medida a realçar é a motivação dos profissionais de saúde no ativo a aumentar a produtividade e a capacidade de resposta dos CSP, pelo incentivo à transição de um modelo de remuneração A (sem incentivos financeiros), para o modelo B (com incentivos financeiros). Criaram-se no entanto, mais uma vez, barreiras à implementação da medida com a definição de um número máximo de Unidades de Saúde que devem proceder a esta transição (Despacho No. 6739-A/2016, de 20 Maio) (Augusto e Fronteira, 2006) ${ }^{\text {a }}$.

Será que o aumento da despesa associada à medida é sustentável?

\section{AUMENTO DE RECURSOS TECNOLÓGICOS/ MATERIAIS /EQUIPAMENTOS/ INSTALAÇÕES NOS CSP}

Ultimamente tem havido uma forte aposta do Governo no sentido do desenvolvimento de recursos tecnológicos na Saúde.

Durante o ano de 2016, o Governo criou o Sistema Integrado de Gestão do Acesso (SIGA) (SNS, 2016) a e pretende continuar a consolidar a aposta neste sistema para o próximo ano, conforme indica no OE 2017 (República Portuguesa, 2016)a .

O SIGA do Serviço Nacional de Saúde (SIGA SNS) pretende assegurar o acesso equitativo às instituições do SNS, no menor espaço de tempo (SNS, 2016)a, nomeadamente aos CSP. Este sistema, atualmente em fase de implementação, reunirá informação do "Sistema Integrado de Gestão de Inscritos para Cirurgia (SIGIC), do Sistema de Referenciação para Primeira Consulta de Especialidade Hospitalar - designado por Consulta a Tempo e Horas (CTH), do aplicativo da Rede Nacional de Cuidados Continuados Integrados (RNCCI), de acesso a outras áreas da prestação de cuidados que não eram monitorizadas, como sejam os Serviços de Urgência, os Meios Complementares de Diagnóstico e Terapêutica (MCDT), as Consultas Subsequentes de Especialidade Hospitalar, os Cuidados de Saúde Primários", entre outras (SNS, 2016a).

Este é portanto um sistema de informação que permite alargar a capacidade dos CSP darem resposta às necessidades da comunidade. É fundamental que a MGF esteja provida de informação sobre o tempo de espera para o acesso a outros níveis de cuidados, sempre que 
Working Paper

Departamento de Ciências Económicas e Empresariais

Universidade Autónoma de Lisboa

necessita de referenciar um paciente para a especialidade ou para os cuidados continuados, e sempre que solicita a execução de exames, agora possíveis em qualquer organização pertencente ao SNS no território nacional, por iniciativa do atual Ministro.

O SIGA está associado ao Portal do SNS, lançado em 2012. Este portal é uma Plataforma de Dados da Saúde que engloba quatro áreas principais: SNS, Institucional, Cidadão e Profissional e que tem inúmeras valências para cidadãos e profissionais de saúde, uma vez que permite o registo e partilha de informação clínica entre o utente, profissionais de saúde e entidades prestadoras de serviços de Saúde (SPMS, 2016), e informação sobre os tempos de espera, que o Governo pretende que seja disponibilizada em tempo real, em todos os serviços de saúde, no médio prazo.

$\mathrm{Na}$ área dirigida aos cidadãos, destacam-se os serviços de marcação de consultas online e de renovação de medicação, isto é, pedir a renovação de receitas sem ter que ir ao centro de saúde

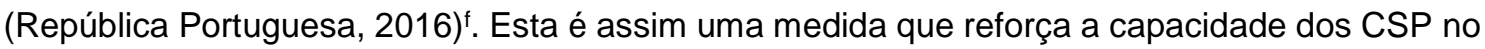
atendimento de outros utentes que necessitam de cuidados e não apenas de receituário.

O Governo apostou também no Sistema Nacional de Avaliação em Saúde (SINAS) (SNS, 2016) Este sistema tem grande importância para que se perceba o que corre bem, nomeadamente nos CSP do país, e para que se possa agir rapidamente perante indicadores cujos resultados não estejam a corresponder a metas previamente definidas. Recorda-se que é importantíssimo monitorizar mas que também é preciso agir em função dos resultados medidos. Sugere-se assim um modelo de descentralização e de autonomia de ação a nível local, para maior celeridade do processo de controlo e reforço da capacidade dos ACES e ULS. Os resultados destas ações devem ser atentamente controlados pela ARS correspondente e, a nível nacional, pela ACSS.

O Governo tem ainda vindo a apostar na aproximação do sistema de saúde aos cidadãos através do desenvolvimento de iniciativas de ordem tecnológica no âmbito dos Programas Nacional de Saúde Infantil e Juvenil e Nacional de Vacinação. Referimo-nos aos projetos Nascer Utente, Notícia Nascimento, eBoletim de Saúde Infantil e Juvenil, e eBoletim de Vacinas, por exemplo. (República Portuguesa, 2016)j.

Por último, foi lançada a aplicação móvel MySNS, que permite aceder, de forma fácil e intuitiva, aos serviços digitais da saúde através dos telemóveis, medida que nos parece apropriada nomeadamente para aproximação do SNS aos adolescentes, caso haja uma boa divulgação da aplicação nas escolas secundárias.

"Esta primeira versão permite acompanhar as notícias do Serviço Nacional de Saúde, aceder a informações úteis para o utente e receber notificações gerais, incluindo questões de saúde pública. Disponibiliza um acesso rápido à Linha de Saúde 24 onde, diariamente, poderá contactar 
Working Paper

Departamento de Ciências Económicas e Empresariais

Universidade Autónoma de Lisboa

com profissionais de saúde qualificados e habilitados, que fornecem os melhores conselhos sobre a forma de lidar com a sua situação de saúde, em particular". (DGS, 2016)a

Quanto a recursos em termos de instalações, na sequência da apresentação do OE 2017, o Governo assumiu a construção de 34 centros de saúde, no âmbito dos protocolos assinados este ano. Destes, 23 serão na região de Lisboa e Vale do Tejo, oito na região centro, um no norte, um no Algarve e um no Alentejo (República Portuguesa, 2016) ${ }^{b}$, estando reunidas as condições para um reforço da capacidade dos CSP nos próximos anos, logo que estas novas instalações tenham os equipamentos, materiais e profissionais adequados à prossecução dos seus objetivos.

Quanto a recursos materiais e equipamentos, o Estado planeou o aumento da despesa com medicamentos e MCDT no OE 2017 (República Portuguesa, 2016)a , sugerindo que vai haver um aumento da capacidade dos CSP neste âmbito.

\section{ESTABELECIMENTO DE ESTRATÉGIAS PARA MAIOR CAPACIDADE DE RESPOSTA}

O Governo assinou no início do ano a Portaria dos Transportes Não Urgentes de Doentes. Esta Portaria tem como objetivo aumentar o acesso aos diferentes níveis de cuidados. Os doentes sujeitos a tratamentos prolongados, independentemente da condição económica, vão deixar de pagar pelo transporte não urgente e o número mínimo de deslocações necessário para haver comparticipação no pagamento vai ser eliminado (oito deslocações, num período de 30 dias). "É eliminado o pagamento para os doentes com incapacidade igual ou superior a $60 \%$ e com insuficiência económica, independentemente de o transporte se destinar à realização de cuidados originados por essa incapacidade. Os doentes oncológicos ou transplantados, bem como insuficientes renais crónicos que realizam diálise peritoneal ou hemodiálise domiciliária, passam a estar também isentos desse pagamento, independentemente do transporte se destinar à realização de atos clínicos inerentes à sua condição. Ficam ainda abrangidas as crianças e adolescentes com doença limitante ou ameaçadora da vida, em caso de insuficiência económica" (República Portuguesa, 2016)i. Esta medida sugere portanto um aumento da capacidade do SNS, e dos CSP, no alcance de mais pessoas e de maior proximidade com a população.

Será que o SNS produz receita suficiente para financiar este acréscimo de despesa? Não será conveniente e pertinente para o SNS suportar apenas o transporte dos mais carenciados?

O OE 2017 informa ainda que haverá para o próximo ano um alargamento da intervenção das equipas de saúde familiar (República Portuguesa, 2016)a , proporcionando também uma maior capacidade de resposta aos CSP. Fica por saber onde, para que patologias e para alcance de que metas. 
Working Paper

Departamento de Ciências Económicas e Empresariais

Universidade Autónoma de Lisboa

Outro exemplo a enaltecer, propício ao aumento da capacidade de resposta dos CSP, é o apoio complementar das autarquias aos CSP. Vejamos o exemplo da Unidade de Saúde Pública da Unidade Local de Saúde de Matosinhos em parceria com a Câmara Municipal de Matosinhos. Estas entidades promoveram um encontro científico "Sinergias em Saúde", onde partilharam ideias e debateram soluções para a literacia em saúde, a promoção de uma vida saudável, a nutrição, a atividade física, a saúde ambiental, o planeamento e a avaliação do impacto das políticas de urbanismo e da mobilidade ativa de pessoas (DGS, 2016) ${ }^{\mathrm{b}}$.

\section{A OPINIÃO DOS PROFISSIONAIS}

Tentou-se perceber a opinião de profissionais que trabalham ativamente em CSP e concluiu-se que tem havido uma melhoria significativa na capacidade de resposta das Unidades Funcionais dos ACES / ULS, nos últimos tempos, relativamente a:

1) Melhoria das infra-estruturas e dos equipamentos disponíveis;

2) Maior possibilidade de contratar médicos, que levou a uma redução do número de pacientes sem médico de família;

3) Redução do horário de trabalho para as $7 \mathrm{~h} /$ diárias levou a um défice no atendimento que foi resolvido por aumento de horas extraordinárias, agora com possibilidade de serem pagas aos médicos dos respetivos ACES / ULS (e não a médicos de empresas privadas);

4) Mobilidade de psicólogos para a Unidade Funcional dos inquiridos, que permitiram reduzir o tempo entre consultas e o tempo de espera para a primeira consulta na doença mental;

5) Há ACES que têm as equipas de ambulatório do Hospital de referência a trabalhar nas suas instalações. Esta sinergia entre cuidados primários e secundários permite uma maior proximidade da comunidade por parte dos especialistas e uma maior articulação com o internamento do hospital, uma vez que os colaboradores do Hospital destacados para as instalações dos ACES / ULS conhecem os outros trabalhadores do Hospital e usam muitas vezes o contacto pessoal para maior eficiência da rede. Estas "equipas hospitalares comunitárias" são financiadas pelas autarquias e funcionam muito bem, de acordo com os profissionais entrevistados;

6) Para melhorar o serviço de Saúde Visual, está a decorrer um projeto de rastreio da retinopatia diabética, financiado pela ARS. Neste caso, o Centro de Saúde tem apenas de selecionar e de divulgar quais os pacientes com maiores necessidades;

7) A capacidade de resposta na Unidade de Ginecologia e Unidade Materno-Infantil é boa. Há uma boa prática de articulação entre os cuidados primários e os secundários na resposta relativa a estas Unidades. 
A título de exemplo, uma das Unidades Funcionais fez, este ano, um protocolo com o Hospital de zona para melhoria da prevenção do cancro do colo do útero. Neste protocolo, a Unidade Funcional faz as citologias em meio líquido que chegam para análise ao Hospital por intermédio de um estafeta financiado pela Unidade.

De salientar ainda que o Hospital tem muitas vezes dificuldade em realizar o rastreio bioquímico e a ecografia obstétrica do primeiro trimestre de gravidez, num período de tempo adequado entre os dois procedimentos. Para a melhoria da resposta em tempo útil, a Unidade Funcional controla e assegura a resposta quando o hospital falha. Esta relação entre níveis de cuidados é sustentada por reuniões mensais entre as Unidades Funcionais dos ACES e o Hospital de referência, na presença das assistentes sociais, para avaliação de soluções face às necessidades prioritariamente identificadas na população e às necessidades de recursos das organizações prestadoras de cuidados.

Há ainda, no entanto, identificação de graves lacunas nos CSP, conforme se descreve:

1) Há ainda falta de nutricionistas, de psicólogos, de assistentes sociais, de higienistas orais, de enfermeiros e de médicos;

2) É preciso trabalhar em mais protocolos e processos assistenciais integrados, como no caso da oncologia;

3) É preciso melhorar a comunicação permitida pelo sistema informático para referenciação de pacientes;

4) Há ACES sem serviços de oftalmologia e que não foram abrangidas pelo rastreio de retinopatia da diabetes prometida pela ARS correspondente;

5) Por último, a maioria dos ACES não tem resposta ao nível da Medicina Física e de Reabilitação e está dependente da capacidade de resposta do Hospital, que é, a maior parte das vezes, deficitária. Para se conseguir tratar estes doentes, o ACES financia os Centros Convencionados Privados que prestam este serviço. Contudo, os ACES/ ULS não têm capacidade para avaliar a evolução do tratamento dos pacientes nem para controlar a prescrição de novos tratamentos, limitando-se a pagar o serviço. De acordo com as fontes, é muitas vezes solicitado ao Hospital a avaliação de pacientes a serem tratados ao abrigo da Convenção há mais de 120 dias, mas o Hospital muitas vezes não dá resposta.

\section{CONCLUSÃO}

Em conclusão, está previsto para o próximo ano um reforço da capacidade dos CSP através do apoio complementar de mais médicos de MGF, na aquisição de medicamentos e de MCDT. Constatou-se que tem havido um esforço na capacitação de recursos para a psicologia e para a saúde visual, embora a maior parte das Unidades Funcionais dos ACES e ULS não possuam assistência em oftalmologia. 
Embora não se tenha encontrado informação acerca do aumento do investimento na obstetrícia e pediatria, considera-se que estas especialidades têm muito bons resultados nos CSP.

Considera-se que a especialidade com necessidade de ação e de investimento prioritário é a Medicina Física e de Reabilitação, por motivo do elevado impacto desta especialidade no orçamento dos ACES / ULS e por motivo da incapacidade de resposta por parte dos CSP do SNS relativamente a esta área. Não se encontrou nenhuma informação sobre o que o Ministro propõe para a resolução deste problema em concreto, provavelmente pela contratação de fisioterapeutas, fisiatras e aquisição de equipamentos adequados.

\section{REFERÊNCIAS BIBLIOGRÁFICAS}

ACSS (2016). Contratação ou renovação de contratos de trabalho pelos hospitais, centros hospitalares e Unidades Locais de Saúde com a natureza de Entidade Pública Empresarial integrados no Serviço Nacional de Saúde - regime excecional previsto no Despacho n.ํ5911c/2016, de 3 de Maio. Lisboa: ACSS. Recuperado em 5 de Outubro de 2016, de http://www. acss.min-saude.pt/Portals/0/Circular\%20N\%C2\%BA\%2020.2016\%20(06.05.2016).pdf

Augusto, G. e Fronteira, I. (2016) 5 - Section 5.3. Recuperado a 5 de Outubro de 2016, de http://www.hspm.org/ countries/portugal25062012/countrypage.aspx

Augusto, G., Simões, J. e Fronteira, I. (2016). New tender for GPs in Portugal. Online HiT, Chapter 5 - Section 5.3. Recuperado a 5 de Outubro de 2016, de http://www.hspm.org/ countries/portugal25062012/countrypage.aspx

DGS (2016)a ${ }^{a}$ SNS em nova aplicação móvel. Recuperado em 5 de Outubro de 2016, de https://www.dgs.pt/em-destaque/sns-em-nova-aplicacao-movel-.aspx

DGS (2016) b. Matosinhos promove Sinergias em Saúde. Recuperado em 5 de Outubro de 2016, de https://www.dgs.pt/em-destaque/matosinhos-promove-sinergias-em-saude.aspx

República Portuguesa (2016)a. Relatório do Orçamento de Estado 2017. Recuperado em 19 de Outubro de 2016, de http://app.parlamento.pt/webutils/docs/doc.pdf?path=61485 23063446f764c3246795a5868774d546f334e7a67774c336470626d6c7561574e7059585270 646d467a4c31684a53556b76644756346447397a4c33427762444d334c56684a53556c664d 6a49756347526d\&fich=ppl37-XIII_22.pdf\&Inline=true

República Portuguesa (2016) ${ }^{\text {b }} 34$ Novos Centros de Saúde em 2017. Recuperado em 20 de Outubro de 2016, de http://www.portugal.gov.pt/pt/ministerios/ms/noticias/ 20161019-msar.aspx

República Portuguesa $(2016)^{c}$. Novas medidas para um SNS de qualidade, acessível e inclusivo. Recuperado em 5 de Outubro de 2016, de http://www.portugal.gov.pt/pt/ ministerios/ms/noticias/20160915-ms-cm-sns.aspx 
República Portuguesa (2016) d. Relançamento da Reforma Cuidados Saúde Primários Plano Estratégico e Operacional. Recuperado em 5 de Outubro de 2016, de http://www.portugal.gov.pt/media/18583832/20160224-seas-ref-cuidados-saudeprimarios.pdf

República Portuguesa (2016)e . SNS integra cerca de 3500 médicos na formação de especialistas. Recuperado em 5 de Outubro de 2016, de http://www.portugal.gov.pt/pt/ministerios/ms/noticias/20160105-ms-medicos-sns.aspx

República Portuguesa $(2016)^{\dagger}$. SNS: Não podemos confundir falta de recursos com falta de organização. Recuperado em 5 de Outubro de 2016, de http://www.portugal .gov.pt/pt/ministerios/ms/noticias/20160201-ms-portal-sns.aspx

República Portuguesa (2016) ${ }^{9}$. Reforma dos cuidados de saúde primários começa por ter mais médicos de família. Recuperado em 5 de Outubro de 2016, de http://www.portugal.gov.pt/pt/ministerios/ms/noticias/20160224-ms-cuidados-primarios.aspx

República Portuguesa (2016) . Não entraremos no Verão com dificuldades inaceitáveis na saúde no Algarve. Recuperado em 5 de Outubro de 2016, de http://www.portugal.gov.pt /pt/ministerios/ms/noticias/20160311-ms-algarve.aspx

República Portuguesa (2016)i. Governo reduz encargos dos doentes em transportes para tratamentos. Recuperado em 5 de Outubro de 2016, de http://www.portugal.gov. $\mathrm{pt} / \mathrm{pt} / \mathrm{ministerios} / \mathrm{ms} /$ noticias/20160406-ms-transporte-doentes.aspx

República Portuguesa (2016)i. Bons resultados e equilíbrio financeiro são compatíveis com as reformas prosseguidas na saúde. Recuperado em 5 de Outubro de 2016, de http://www.portugal.gov.pt/pt/ministerios/ms/noticias/20160525-ms-ar.aspx

SNS (2016)a. SIGA SNS - Sistema Integrado de Gestão do Acesso no Serviço Nacional de Saúde. Recuperado em 5 de Outubro de 2016, de https://www.sns.gov.pt/sns/sistema-integradode-gestao-do-acesso-no-servico-nacional-de-saude-siga-sns/

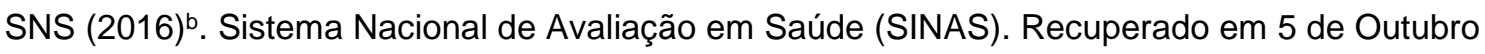
de 2016, de https://www.sns.gov.pt/home/sistema-nacional-de-avaliacao-em-saude-sinas/

SPMS (2016). PDS - Plataforma de Dados da Saúde. Lisboa: Serviços Partilhados do Ministério da Saúde. Recuperado em 5 de Outubro de 2016, de http://spms.min-saude.pt/product/portaldo-utente/ 